\title{
SRB MEASURES FOR WEAKLY EXPANDING MAPS
}

\author{
VILTON PINHEIRO
}

\begin{abstract}
We construct SRB measures for endomorphisms satisfying conditions far weaker than the usual non-uniform expansion. As a consequence, the definition of a non-uniformly expanding map can be weakened. We also prove the existence of an absolutely continuous invariant measure for local diffeomorphisms, only assuming the existence of hyperbolic times for Lebesgue almost every point in the manifold.
\end{abstract}

\section{INTRODUCTION}

In [11, Keller proved the existence of an absolutely continuous invariant measure for any non-flat multimodal map $f$, whenever it satisfies the negative Schwarzian derivative condition and there exists a constant $\lambda$ such that for Lebesgue almost all $x$

$$
\limsup _{n \rightarrow \infty} \frac{1}{n} \sum_{j=0}^{n-1} \log \left|D f\left(f^{j}(x)\right)\right| \geq \lambda>0 .
$$

Besides that, Keller showed the existence of a finite number of these measures whose union of basins contains Lebesgue almost all point of the domain of $f$. This measure is called a physical or SRB (SinaiRuelle-Bowen) measure.

The result of Keller was somewhat generalized by Alves-BonattiViana [4] in the context of non-flat maps defined on a compact Riemannian manifold $M$. For this, the distortion control condition of negative Schwarzian derivative was replaced by the so called slow recurrence to the critical set and it was assumed that

$$
\liminf _{n \rightarrow \infty} \frac{1}{n} \sum_{j=0}^{n-1} \log \left(\left\|\left(D f\left(f^{j}(x)\right)\right)^{-1}\right\|^{-1}\right) \geq \lambda>0
$$

for Lebesgue almost all $x \in M$. Of course, if the dimension of $M$ is one we have $|D f(x)|=\left\|(D f(x))^{-1}\right\|^{-1}$. Observe that $\left\|(D f(x))^{-1}\right\|^{-1}>1$

Date: November 7, 2018.

Work carried out at the Federal University of Bahia (Brazil) and ICTP (Italy) Partially supported by PADCT/CNPq(Brazil). 
means that $D f(x)$ expands in all directions, that is, $\|(D f(x)) v\| \geq$ $\gamma\|v\| \forall v \in T_{x} M$ (where $\left.\gamma=\left\|(D f(x))^{-1}\right\|^{-1}\right)$. Systems satisfying (2) are called non-uniformly expanding, they generalize the uniformly expanding systems. As particular examples of this kind of systems we can mention one-dimensional maps with positive Lyapunov exponents (like quadratic maps and, in general, non-flat multimodal maps [8]), and in higher dimension, the Viana maps [13.

We note that any multimodal map that satisfies Keller's hypothesis also satisfies the slow recurrence condition to the critical set. That is, if $f$ is a $C^{3}$ non-flat multimodal map with positive Lyapunov exponent (i.e., it satisfies (11)) then the hypothesis of negative Schwarzian derivative is stronger than the condition of slow recurrence to the critical set (see Proposition 2.2).

The purpose of this paper is to generalize the result of Keller in the context of [4] (replacing the negative Schwarzian derivative by the slow recurrence to the critical set) with the far more weak condition of lim sup as it appears in Keller's theorem, i.e.,

$$
\limsup _{n \rightarrow \infty} \frac{1}{n} \sum_{j=0}^{n-1} \log \left(\left\|\left(D f\left(f^{j}(x)\right)\right)^{-1}\right\|^{-1}\right) \geq \lambda>0 .
$$

Moreover, we prove that the condition (3) indeed implies (21) and so, the definition of non-uniformly expanding map can be weakened.

1.1. Statement of results. Let $M$ be a compact Riemannian manifold of dimension $d \geq 1$ and Leb a normalized Riemannian volume form on $M$ that we call Lebesgue measure.

A map $f: M \rightarrow M$ will be called non-flat if $f$ is local $C^{2}$ diffeomorphism in the whole manifold except in a non-degenerate critical (or singular) set $\mathcal{C} \subset M$. The definition of non-degenerate critical set is given at the beginning of section 2 (If $\operatorname{dim}(M)=1$ and $f$ satisfies the usual definition of non-flatness (see [8]), then it also satisfies the definition given above).

We say that $f$ satisfies the slow approximation condition if given any $\epsilon>0$ there exists $\delta>0$ such that for Lebesgue almost every point $x \in M$ we have

$$
\limsup _{n \rightarrow+\infty} \frac{1}{n} \sum_{j=0}^{n-1}-\log \operatorname{dist}_{\delta}\left(f^{j}(x), \mathcal{C}\right) \leq \epsilon,
$$

where $\operatorname{dist}_{\delta}(x, \mathcal{C})$ denotes the $\delta$-truncated distance from $x$ to $\mathcal{C}$ defined as $\operatorname{dist}_{\delta}(x, \mathcal{C})=\operatorname{dist}(x, \mathcal{C})$, if $\operatorname{dist}(x, \mathcal{C}) \leq \delta$, and $\operatorname{dist}_{\delta}(x, \mathcal{C})=1$, otherwise. 
We call the basin of some invariant measure $\nu$ the set $\mathcal{B}(\nu)$ of the points $x \in M$ such that the average of Dirac measures along the orbit of $x$ converges in the weak ${ }^{*}$ topology to $\nu$, that is,

$$
\lim _{n \rightarrow+\infty} \frac{1}{n} \sum_{j=0}^{n-1} \phi\left(f^{j}(x)\right)=\int \phi d \nu, \forall \phi \in C^{0}(M) .
$$

Theorem 1. Let $f: M \rightarrow M$ be a non-flat map satisfying the slow approximation condition. If $f$ (or some fixed iterate) satisfies

$$
\liminf _{n \rightarrow \infty} \frac{1}{n} \sum_{i=0}^{n-1} \log \left(\left\|\left(D f\left(f^{i}(x)\right)\right)^{-1}\right\|\right) \leq-\lambda<0
$$

(or equivalently satisfies (3)) for Lebesgue almost every point $x \in M$, then there exists a finite collection of ergodic absolutely continuous invariant measures such that almost every point in $M$ belongs to the basin of one of these measures.

Theorem 1 is in fact a consequence of the Main Theorem below that guarantees the existence of a global Markov structure with integrable time function. A global Markov structure is composed by a time function $x \mapsto R(x) \in \mathbb{N} \cup\{\infty\}$, an induced map $F(x)=f^{R(x)}(x)$ defined almost everywhere and a countable partition refining a finite triangulation of $M$. Each element of this partition is sent by $F$, with good properties (see section 3.3 for details), onto an element of the triangulation. One can prove that such $F$ has an absolutely continuous invariant measure $\nu$. Moreover, whenever $R$ is $\nu$-integrable, it generates an absolutely continuous $f$-invariant finite measure. We observe that the existence of a Markov structure allows a more deep study of the dynamical properties of the map $f$. For instance, it was used in [5, 7, 9, 14, 15] to study the decay of correlations and prove the Central Limit Theorem for a large class of maps.

Main Theorem. Every map satisfying the hypothesis of theorem 1 has a global Markov Structure with integrable time function.

The proof of the main theorem is a mix of the strategy adopted by Alves-Luzzatto-Pinheiro in [5] for non-uniform expanding maps (inspired in Young's paper [14) and the proof of Keller's theorem in 8]. Here we are able to simplify the proof of the existence of the Markov Structure that appears in [5, 9, 14] and make it closer to the one dimensional case.

A map $f: M \rightarrow M$ is called non-uniformly expanding if $f$ is a nonflat map satisfying the slow approximation condition and it (or some 
fixed iterate) satisfies, for Lebesgue almost every point $x \in M$, the following condition,

$$
\limsup _{n \rightarrow \infty} \frac{1}{n} \sum_{i=0}^{n-1} \log \left(\left\|\left(D f\left(f^{i}(x)\right)\right)^{-1}\right\|\right) \leq-\lambda<0
$$

(or equivalently satisfies (21)).

Theorem 2. A $C^{2}$ endomorphism is non-uniformly expanding if and only if it satisfies the hypothesis of theorem 1 .

We want to remark that Theorem 2 deals with a more restricted class of maps than Theorem 1. For instance, maps with a singular set $\mathcal{S}\left(|\operatorname{det} D f|_{\mathcal{S}} \mid=+\infty\right)$ are allowed in the hypothesis of Theorem 1 . This difference happens because, in the proof of Theorem 2 , we need a $C^{2}$ map to guarantee the integrability of $\log \left\|(D f)^{-1}\right\|$ with respect to any absolutely continuous invariant measure (see Lemma 4.2).

A crucial ingredient in the proof of these results is the existence, for almost all point $x \in M$, of moments $n=n(x) \in \mathbb{N}$ such that $f$ looks like an uniformly expanding map on some neighborhood $V_{n}(x)$ of $x$, that is, this neighborhood is sent by $f^{n}$, with good properties of expansion and distortion, to some big ball $B\left(f^{n}(x)\right)$ centered at $f^{n}(x)$. The last result of this paper is about hyperbolic times which are associated with these moments of expansion mentioned above (see Proposition 2.5). In 3] Alves-Araújo proved the existence of an $S R B$ measure when the critical set is empty and the first hyperbolic time is Lebesgue integrable. Here, we were able to remove the hypothesis of integrability.

Theorem 3. Let $f: M \rightarrow M$ be a $C^{2}$ covering map (local diffeomorphism) on a compact manifold $M$. If the first hyperbolic time function is defined for Lebesgue almost every point of $M$, then $f$ is a non-uniformly expanding map.

\section{Hyperbolic Times}

Definition 2.1. Let $f: M \rightarrow M$ be a $C^{2}$ local diffeomorphism in the whole manifold except in a critical (or singular) set $\mathcal{C} \subset M$. We say that $\mathcal{C} \subset M$ is a non-degenerate critical set, more precisely, a $\beta$-nondegenerate critical set $(\beta>0)$ if $\exists B>0$ such that the following three conditions hold:

$$
\begin{aligned}
& \frac{1}{B} \operatorname{dist}(x, \mathcal{C})^{\beta} \leq \frac{\|D f(x) v\|}{\|v\|} \leq B \operatorname{dist}(x, \mathcal{C})^{-\beta} \text { for all } x \in M \backslash \mathcal{C} \\
& \text { and } v \in T_{x} M .
\end{aligned}
$$

For every $x, y \in M \backslash \mathcal{C}$ with $\operatorname{dist}(x, y)<\operatorname{dist}(x, \mathcal{C}) / 2$ we have 


$$
\begin{aligned}
& |\log |\left|D f(x)^{-1}\right|\left|-\log \left\|D f(y)^{-1}\right\|\right| \leq \frac{B}{\operatorname{dist}(x, \mathcal{C})^{\beta}} \operatorname{dist}(x, y) \\
& |\log | \operatorname{det} D f(x)|-\log | \operatorname{det} D f(y)|| \leq \frac{B}{\operatorname{dist}(x, \mathcal{C})^{\beta}} \operatorname{dist}(x, y) .
\end{aligned}
$$

Proposition 2.2. Let $f: I \rightarrow I$ be a non-flat $C^{3}$ multimodal map of the interval I with positive Lyapunov exponent, i.e., it satisfies (1) for almost every point $x \in I$. If $f$ satisfies the negative Schwarzian derivative condition, then it also satisfies the condition of slow recurrence to the critical set.

Proof. By Keller's result, there exists a finite collection $\mu_{1}, \ldots, \mu_{s}$ of ergodic absolutely continuous invariant measures such that Leb(I) $\left.\mathcal{B}\left(\mu_{1}\right) \cup \ldots \cup \mathcal{B}\left(\mu_{s}\right)\right)=0$. Moreover, the support of each $\mu_{j}$ is a finite union of intervals and for Lebesgue almost all $p \in \mathcal{B}\left(\mu_{j}\right)$ there is some $n_{0}=n_{0}(p)$ such that $q=f^{n_{0}}(p)$ is a $\mu_{j}$-generic point, in particular $q \in \operatorname{supp} \mu_{j}$. As $f$ is $C^{3}$, there are constants $A_{0}, A_{1}>0$ such that $|D f(x)|<A_{0} \operatorname{dist}(x, \mathcal{C})<A_{1}, \forall x \in I$. On the other hand, as $\log |D f| \in L^{1}\left(\mu_{j}\right)$ (Remark 1.2 of [12]), it follows that $\varphi: x \mapsto$ $\log \operatorname{dist}(x, \mathcal{C})$ is $\mu_{j}$-integrable.

Taking $I_{\delta}=\{x \in I \| \operatorname{dist}(x, \mathcal{C})<\delta\}$, we have $\operatorname{Leb}\left(I_{\delta}\right) \searrow 0$. So, $\mu_{j}\left(I_{\delta}\right) \rightarrow 0$ and also $\lim _{\delta \rightarrow 0} \int \varphi_{\delta} d \mu_{j}=\lim _{\delta \rightarrow 0} \int_{I_{\delta}} \varphi d \mu_{j}=0$, where $\varphi_{\delta}=\log \operatorname{dist}_{\delta}(\cdot, \mathcal{C})$. Thus, given $\varepsilon>0$ let $\delta>0$ be such that $-\int \varphi_{\delta} d \mu_{j}$ $\leq \varepsilon, \forall j=1, \ldots, s$. By Birkhoff's ergodic theorem,

$$
\lim \frac{1}{n} \sum_{j=0}^{n-1}-\log \operatorname{dist}_{\delta}\left(f^{j}(q), \mathcal{C}\right)=-\int \varphi_{\delta} d \mu_{j} \leq \varepsilon
$$

for any $\mu_{j}$-generic $q$. Thus,

$$
\begin{gathered}
\lim \frac{1}{n} \sum_{j=0}^{n-1}-\log \operatorname{dist}_{\delta}\left(f^{j}(p), \mathcal{C}\right)= \\
=\lim \frac{1}{n} \sum_{j=0}^{n-1}-\log \operatorname{dist}_{\delta}\left(f^{j}\left(f^{n_{0}(p)}(p)\right), \mathcal{C}\right) \leq \varepsilon,
\end{gathered}
$$

for Lebesgue almost all $p \in \mathcal{B}\left(\mu_{1}\right) \cup \ldots \cup \mathcal{B}\left(\mu_{s}\right)$.

Let us fix $0<b<\frac{1}{2} \min \{1,1 / \beta\}$. If $f: M \rightarrow M$ is a $C^{2}$ local diffeomorphism outside a $\beta$-non-degenerate critical set $\mathcal{C}$ then, given $0<\sigma<1$ and $\delta>0$, we will say that $n$ is a $(\sigma, \delta)$-hyperbolic time for a point $x \in M$ if for all $1 \leq k \leq n$ we have $\prod_{j=n-k}^{n-1}\left\|\left(D f \circ f^{j}(x)\right)^{-1}\right\| \leq$ $\sigma^{k}$ and $\operatorname{dist}_{\delta}\left(f^{n-k}(x), \mathcal{C}\right) \geq \sigma^{b k}$. We denote the set of points of $M$ such that $n \in \mathbb{N}$ is $(\sigma, \delta)$-hyperbolic time by $H_{n}(\sigma, \delta)$. 
Proposition 2.3. 4] Let $f: M \rightarrow M$ be a $C^{2}$ non-flat map satisfying the slow approximation condition. Given $\lambda>0$ there exist $\delta>0$ and $\theta>0$, depending only on $f$ and $\lambda$, such that

$$
\#\left\{1 \leq j \leq n \| x \in H_{j}\left(e^{-\lambda / 4}, \delta\right)\right\} \geq \theta n,
$$

whenever $\sum_{i=0}^{n-1} \log \left\|\left(D f\left(f^{i}(x)\right)\right)^{-1}\right\|^{-1}>\lambda n$.

Corollary 2.4. Let $f: M \rightarrow M$ be a $C^{2}$ non-flat map satisfying the slow approximation condition. Given $\lambda>0$ there exist $\delta>0$ and $\theta>0$, depending only on $f$ and $\lambda$, such that if $A \subset M$ with $\limsup \frac{1}{n} \sum_{i=0}^{n-1} \log \left\|\left(D f\left(f^{i}(x)\right)\right)^{-1}\right\|^{-1}>\lambda$ for Lebesgue almost all $x \in$ A then

$$
\limsup _{n} \frac{1}{n} \#\left\{1 \leq j \leq n \| x \in H_{j}\left(e^{-\lambda / 4}, \delta\right)\right\} \geq \theta
$$

for Lebesgue almost all $x \in A$

We finish this section stating a proposition that assures a good behavior, with respect to $f^{n}$, of a neighborhood of a point $x$ when $n$ is a hyperbolic time for this point.

Proposition 2.5. Let $f: M \rightarrow M$ be a $C^{2}$ local diffeomorphism outside a non-degenerate critical set $\mathcal{C}$. Given $\sigma<1$ and $\delta>0$, there exist $\delta_{1}, \rho>0$, depending only on $\sigma, \delta$ and on the map $f$, such that for any $x \in M$ and $n \geq 1$ a $(\sigma, \delta)$-hyperbolic time for $x$, there exists a neighborhood $V_{n}(x)$ of $x$ with the following properties:

(1) $f^{n}$ maps $V_{n}(x)$ diffeomorphically onto the ball $B_{\delta_{1}}\left(f^{n}(x)\right)$;

(2) $\operatorname{dist}\left(f^{n-j}(p), f^{n-j}(q)\right) \leq \sigma^{j / 2} \operatorname{dist}\left(f^{n}(p), f^{n}(q)\right) \forall p, q \in V_{n}(x)$ and $1 \leq j<n$;

(3) $\log \left|\frac{\operatorname{det} D f^{n}(p)}{\operatorname{det} D f^{n}(q)}\right| \leq \rho \operatorname{dist}\left(f^{n}(p), f^{n}(q)\right) \forall p, q \in V_{n}(x)$.

We shall often refer to the sets $V_{n}(x)$ as hyperbolic pre-balls and to their images $f^{n}\left(V_{n}(x)\right)$ as hyperbolic balls. Notice that the latter are indeed balls of radius $\delta_{1}$.

Proof. For the proofs of items 1 and 2 see Lemma 5.2 in 44. Let $p, q \in V_{n}(x)$. As $n$ is a hyperbolic time for $x$, it follows from item (2) above that $\operatorname{dist}\left(f^{j}(q), \mathcal{C}\right) \geq \operatorname{dist}\left(f^{j}(x), \mathcal{C}\right)-\operatorname{dist}\left(f^{j}(x), f^{j}(q)\right) \geq$ $\sigma^{b(n-j)}-\delta_{1} \sigma^{(n-j) / 2} \geq\left(1-\delta_{1}\right) \sigma^{b(n-j)}$. Now, using condition (3) of the definition of a non-degenerate critical set, we get

$$
\log \left|\frac{\operatorname{det} D f^{n}(q)}{\operatorname{det} D f^{n}(p)}\right| \leq \sum_{j=0}^{n-1} \log \left|\frac{\operatorname{det} D f\left(f^{j}(q)\right)}{\operatorname{det} D f\left(f^{j}(p)\right)}\right| \leq
$$




$$
\begin{gathered}
\leq \sum_{j=0}^{n-1} B \frac{\sigma^{(n-j) / 2} \operatorname{dist}\left(f^{n}(q), f^{n}(p)\right)}{\left(\left(1-\delta_{1}\right) \sigma^{b(n-j)}\right)^{\beta}} \leq \\
\leq \frac{B}{\left(1-\delta_{1}\right)^{\beta}\left(1-\sigma^{1 / 2-b \beta}\right)} \operatorname{dist}\left(f^{n}(q), f^{n}(p)\right)
\end{gathered}
$$

\section{Markov Structures and The Partitioning Algorithm}

In this section we will say what we mean by a Global Markov Structure and prove the first part of the Main Theorem. A Global Markov Structure for a map $f: M \rightarrow M$ is an induced piecewise uniformly expanding Markovian map defined in an open subset of $M$ with full Lebesgue measure. Precisely, we will say that $f$ has a Global Markov Structure if there exists an open subset $M^{\prime} \subset M$ with $\operatorname{Leb}\left(M \backslash M^{\prime}\right)=0$ and a function $R: M^{\prime} \rightarrow \mathbb{N} \cup\{+\infty\}$ (called time function) such that the induced map $F: M^{\prime} \rightarrow M$ given by $F(x)=f^{R(x)}(x)$ is a piecewise uniformly expanding Markovian map defined on $M$ (see the next paragraph). The pair $(F, R)$ is called a Global Markov Structure for $f$.

3.1. Piecewise expanding Markovian map. $A C^{2}$ piecewise uniformly expanding Markovian map defined on $M$ is a map $F$ defined in an open subset of $M$ with full Lebesgue measure such that there is a $(\bmod 0)$ countable partition $\mathcal{P}^{\prime}$ refining a finite partition $(\bmod 0)$ $\mathcal{P}=\left\{P_{1}, \ldots, P_{n}\right\}$, such that each element is given as the interior of some element in a triangulation $\mathcal{T}$ of $M$, with $F, \mathcal{P}^{\prime}$ and $\mathcal{P}$ satisfying the following properties:

(1) $P_{j}$ has a piecewise $C^{2}$ boundary with finite $(d-1)$-dimensional volume, $\forall j=1, \ldots, n$.

(2) $\exists 0<\kappa<1$ such that $\left\|D F(x)^{-1}\right\|<\kappa, \forall x \in U$ and $\forall U \in \mathcal{P}^{\prime}$.

(3) $\forall U \in \mathcal{P}^{\prime},\left.F\right|_{U}$ is a $C^{2}$ diffeomorphism onto some element of $\mathcal{P}$.

(4) $\exists K>0$ such that $\log \left|\frac{\operatorname{det} D F(x)}{\operatorname{det} D F(y)}\right| \leq K \operatorname{dist}(F(x), F(y)), \forall x, y \in$ $U$ and $\forall U \in \mathcal{P}^{\prime}$.

The theorem below assures that every $C^{2}$ piecewise uniformly expanding Markovian map $F: \Delta \rightarrow \Delta$ has an absolutely continuous invariant measure $\nu$ whose density belongs to $L^{\infty}(L e b)$; see e.g. Lemma 4.4.1 of [1. Moreover, it is straightforward to check that if $R$ is $\nu$ integrable, then

$$
\mu=\sum_{j=0}^{\infty} f_{*}^{j}(\nu \mid\{R>j\})
$$


is an absolutely continuous $f$-invariant measure. Here $\nu \mid\{R>j\}$ denotes the measure given by $\nu \mid\{R>j\}(A)=\nu(A \cap\{R>j\})$, and $f_{*}^{j}$ denotes the push-forward of the measure by $f^{j}$.

We say that the Markov Structure has integrable time function if $R$ is integrable with respect to any absolutely continuous $F$ invariant measure. As a consequence to the theorem below, if we want to show that a Markov Structure has integrable time function, we only need to verify the integrability of $R$ with respect to the finite collection of ergodic absolutely continuous invariant measures given by this theorem.

Theorem 4. 1, 2, 6] If $F: \Delta \rightarrow \Delta$ is a $C^{2}$ piecewise uniformly expanding Markovian map, then there exists a finite set of ergodic absolutely continuous invariant measures such that Lebesgue almost every point in $\Delta$ belongs to the basin of one of these measures. Moreover, the density of each of these measures with respect to Lebesgue is uniformly bounded by some constant.

3.2. The Partitioning Algorithm. Let $f: M \rightarrow M$ be a $C^{2}$ nonflat map satisfying the slow approximation condition and take $\lambda_{0}>0$. Suppose that

$$
\limsup _{n \rightarrow \infty} \frac{1}{n} \sum_{i=0}^{n-1} \log \left\|\left(D f\left(f^{i}(x)\right)\right)^{-1}\right\|^{-1}>\lambda_{0}
$$

for Lebesgue almost every $x \in M$. In this section we will show how one can construct a Markovian Structure on $M$.

Replacing, if is necessary, $f$ by some $f^{n_{0}}$ we may assume, without loss of generality, that $\lambda_{0}>16 \log 2$.

Let $\lambda=\lambda_{0} / 4$ and

$$
\sigma=\exp (-\lambda)<\frac{1}{16}
$$

be fixed from now on. Let $\delta>0$ be given by Proposition 2.3 and let $\delta_{1}>0$ be the radius of the hyperbolic ball given by Proposition 2.5 . For short, let $H_{j}=H_{j}(\sigma, \delta), \forall j \geq 1$.

Remark 3.1. It follows from Corollary 2.4 the existence of a constant $\theta>0$ such that $\limsup _{n} \frac{1}{n} \#\left\{1 \leq j \leq n \| x \in H_{j}\right\} \geq \theta$ for Lebesgue almost all $x \in M$.

Choose some $0<\delta_{0}<\delta_{1} / 4$ and a finite partition $\mathcal{P}$ of $M(\bmod 0)$ generated by the interior of the elements of a triangulation of $M$ with diameter smaller than $\delta_{0}$, that is, diameter $(P)<\delta_{0} \forall P \in \mathcal{P}$.

Given $U \subset M$ and $0<r$ define the $r$-neighborhood of $U$ as $B_{r}(U)=$ $\{x \in M \| \operatorname{dist}(x, U)<r\}$. For each element $P \in \mathcal{P}$, set $P^{1}=B_{\delta_{0}}(P)$ 
and let $\left\{I_{k}^{1}(P)\right\}_{k \in \mathbb{N}}$ be the partition $(\bmod 0)$ of $P^{1} \backslash P$ into the collection of "rings"

$$
I_{k}^{1}(P)=\left\{x \in P^{1} \backslash P \| \delta_{0} \sigma^{k / 2} \leq \operatorname{dist}(x, \partial P)<\delta_{0} \sigma^{(k-1) / 2}\right\},
$$

where $k=1,2,3, \ldots$.

Now we present an algorithm to construct a refinement $\mathcal{P}^{\prime}$ of $\mathcal{P}$ such that each element $Q \in \mathcal{P}^{\prime}$ will be sent, at a hyperbolic time, diffeomorphically onto some element of $\mathcal{P}$. That is, each $Q \in \mathcal{P}^{\prime}$ will be contained in some hyperbolic pre-ball $V_{n}(q)$ for some $q \in Q \cap H_{n}$ and there will be some $P \in \mathcal{P}$ such that $Q=\left(f^{n} \mid V_{n}(q)\right)^{-1}(P)$. The major difficulty of the algorithm is to prevent overlaps of the new elements with the elements constructed in previous steps. To deal with this problem, we will define inductively, for each step $n \in \mathbb{N}$, an auxiliary function $t_{n}$ (we are assuming $0 \in \mathbb{N}$ ). This function will tell us if it is possible to use, at the step $n$, a point $x \in H_{n}$ as the "center" of a hyperbolic pre-ball $V_{n}(x)$ to generate a new element of the partition.

Given an element $P_{0} \in \mathcal{P}$, let us set $\Delta_{0}\left(P_{0}\right)=P_{0}$. We define a function $t_{0}: P_{0} \rightarrow \mathbb{N}$ by

$$
t_{0}(x)=\min \left\{k \in \mathbb{N} \| \delta_{0} \sigma^{k / 2} \leq \operatorname{dist}\left(x, \partial P_{0}\right)\right\},
$$

and the subsets $\mathbb{A}_{0}\left(P_{0}\right)$ and $\mathbb{B}_{0}\left(P_{0}\right)$ of $\Delta_{0}\left(P_{0}\right)$ given by

$$
\begin{aligned}
& \mathbb{A}_{0}\left(P_{0}\right)=\left\{x \in \Delta_{0}\left(P_{0}\right) \| t_{0}(x)=0\right\} \text { and } \\
& \mathbb{B}_{0}\left(P_{0}\right)=\left\{x \in \Delta_{0}\left(P_{0}\right) \| t_{0}(x)>0\right\} .
\end{aligned}
$$

In fact, as diameter $\left(P_{0}\right)<\delta_{0}$, we have $\mathbb{A}_{0}\left(P_{0}\right)=\emptyset$ and $\mathbb{B}_{0}\left(P_{0}\right)=P_{0}$.

We define inductively the sets $\Delta_{n}\left(P_{0}\right), \mathbb{A}_{n}\left(P_{0}\right), \mathbb{B}_{n}\left(P_{0}\right)$ and functions $t_{n}: P_{0} \rightarrow \mathbb{N}$ for every $n \in \mathbb{N}$. For this, let us assume that for some $n>0$ the sets $\Delta_{k}\left(P_{0}\right), \mathbb{A}_{k}\left(P_{0}\right), \mathbb{B}_{k}\left(P_{0}\right)$ and functions $t_{k}: P_{0} \rightarrow \mathbb{N}$ are already defined for all $0 \leq k \leq n-1$.

Our construction will depend on how many points of $\mathbb{A}_{n-1}\left(P_{0}\right)$ have $n$ as a hyperbolic time. So, for the definition of $\Delta_{n}\left(P_{0}\right)$ and $t_{n}$, we consider two cases.

First case. If $\operatorname{Leb}\left(\mathbb{A}_{n-1}\left(P_{0}\right) \cap H_{n}\right)=0$ we set $\Delta_{n}\left(P_{0}\right)=\Delta_{n-1}\left(P_{0}\right)$. Setting $t_{n}^{\prime}: P_{0} \rightarrow \mathbb{N}$ as $t_{n}^{\prime}(x)=0 \forall x$, we define $t_{n}: P_{0} \rightarrow \mathbb{N}$ as

$$
t_{n}(x)=\max \left\{t_{n}^{\prime}(x), t_{n-1}(x)-1\right\}\left(=\max \left\{0, t_{n-1}(x)-1\right\}\right) .
$$

Thus, we may write

$$
t_{n}(x)=\left\{\begin{array}{ll}
0 & \text { if } x \in \mathbb{A}_{n-1}\left(P_{0}\right) \\
t_{n-1}(x)-1 & \text { if } x \in \mathbb{B}_{n-1}\left(P_{0}\right)
\end{array} .\right.
$$


Second case. If $\operatorname{Leb}\left(\mathbb{A}_{n-1}\left(P_{0}\right) \cap H_{n}\right)>0$, then, for Lebesgue almost every point $x \in \mathbb{A}_{n-1}\left(P_{0}\right) \cap H_{n}$, there exists some $P \in \mathcal{P}$ such that $f^{n}(x) \in P$. As the diameter of $P^{1}$ is smaller than $3 \delta_{1} / 4$ we also have $B_{\delta_{1}}\left(f^{n}(x)\right) \supset P^{1} \supset P$. Proposition 2.5 assures that there exists a hyperbolic pre-ball $V_{n}(x)$ such that $f^{n} \mid V_{n}(x): V_{n}(x) \rightarrow B_{\delta_{1}}\left(f^{n}(x)\right)$ is a diffeomorphism with uniform bounded distortion ( not depending on $x$ or $n)$. Denote by $\mathcal{U}_{n}\left(P_{0}\right)$ the collection of all sets $\left(f^{n} \mid V_{n}(x)\right)^{-1}(P)$ contained in $P_{0}$, where $x \in \mathbb{A}_{n-1}\left(P_{0}\right) \cap H_{n}$ and $f^{n}(x) \in P \in \mathcal{P}$.

We claim that the elements of $\mathcal{U}_{n}\left(P_{0}\right)$ are mutually disjoint. To check this, let us assume that $x_{1}, x_{2} \in H_{n} \cap P_{0}, f^{n}\left(x_{1}\right) \in P_{1} \in \mathcal{P}$ and $f^{n}\left(x_{2}\right) \in$ $P_{2} \in \mathcal{P}$. Also, let $U=\left(\left.f^{n}\right|_{V_{n}\left(x_{1}\right)}\right)^{-1}\left(P_{1}\right)$ and $V=\left(\left.f^{m}\right|_{V_{m}\left(x_{2}\right)}\right)^{-1}\left(P_{2}\right)$. As the elements of $\mathcal{P}$ are small compared to $B_{\delta_{1}}\left(f^{n}\left(x_{1}\right)\right)$ or $B_{\delta_{1}}\left(f^{n}\left(x_{2}\right)\right)$ $\left(\operatorname{diameter}(P)<\delta_{0}<\delta_{1} / 4 \forall P \in \mathcal{P}\right)$, if $P_{1}=P_{2}$, then $\left.\left(\left.f^{n}\right|_{V_{n}\left(x_{1}\right)}\right)^{-1}\right|_{P_{1}}=$ $\left.\left(\left.f^{n}\right|_{V_{n}\left(x_{2}\right)}\right)^{-1}\right|_{P_{2}}$ and so, $U=V$. On the other hand, if $P_{0} \neq P_{1}$, then $f^{n}(U) \cap f^{n}(V)=P_{0} \cap P_{1}=\emptyset$ and so, $U \cap V=\emptyset$, proving the claim.

Given $U \in \mathcal{U}_{n}\left(P_{0}\right)$, with $U=\left(f^{n} \mid V_{n}(x)\right)^{-1}(P)$ and $P \in \mathcal{P}$, let

$$
U^{1}=\left(f^{n} \mid V_{n}(x)\right)^{-1}\left(P^{1}\right)
$$

and, for $j=1,2,3, \ldots$,

$$
U^{1}(j)=\left(f^{n} \mid V_{n}(x)\right)^{-1}\left(I_{j}^{1}(P)\right) .
$$

Define

$$
\Delta_{n}\left(P_{0}\right)=\Delta_{n-1}\left(P_{0}\right) \backslash \bigcup_{U \in \mathcal{U}_{n}\left(P_{0}\right)} U .
$$

For each point $x \in \bigcup_{U \in \mathcal{U}_{n}\left(P_{0}\right)} U^{1} \backslash U$, set

$$
t_{n}^{\prime}(x)=\max \left\{i \| x \in U^{1}(i) \text { and } U \in \mathcal{U}_{n}\left(P_{0}\right)\right\},
$$

and for the other points $x \in P_{0}$, set $t_{n}^{\prime}(x)=0$.

As before, define $t_{n}: P_{0} \rightarrow \mathbb{N}$ by

$$
t_{n}(x)=\max \left\{t_{n}^{\prime}(x), t_{n-1}(x)-1\right\} .
$$

Because $t_{n}^{\prime}(x)=0$ for any $x$ outside $\bigcup_{U \in \mathcal{U}_{n}\left(P_{0}\right)} U^{1} \backslash U$, we observe that

$$
t_{n}(x)= \begin{cases}t_{n}^{\prime}(x) & \text { if } x \in \bigcup_{U \in \mathcal{U}_{n}\left(P_{0}\right)} U^{1} \backslash U \\ 0 & \text { if } x \in \mathbb{A}_{n-1}\left(P_{0}\right) \backslash\left(\bigcup_{U \in \mathcal{U}_{n}\left(P_{0}\right)} U^{1} \backslash U\right), \\ t_{n-1}(x)-1 & \text { if } x \in \mathbb{B}_{n-1}\left(P_{0}\right) \backslash\left(\bigcup_{U \in \mathcal{U}_{n}\left(P_{0}\right)} U^{1} \backslash U\right)\end{cases}
$$

whenever $x \in \Delta_{n}\left(P_{0}\right)$. This finishes the second case.

In both cases, $\Delta_{n}\left(P_{0}\right)$ and $t_{n}$ are defined and we set

$$
\mathbb{A}_{n}\left(P_{0}\right)=\left\{x \in \Delta_{n}\left(P_{0}\right) \| t_{n}(x)=0\right\}
$$

and 


$$
\mathbb{B}_{n}\left(P_{0}\right)=\left\{x \in \Delta_{n}\left(P_{0}\right) \| t_{n}(x)>0\right\} .
$$

Remark 3.2. As $t_{n}=\max \left\{t_{n}^{\prime}, t_{n-1}-1\right\} \forall n \geq 1$, we get $t_{n} \geq t_{n-1}-1 \geq$ $t_{n-2}-2 \geq \ldots \geq t_{n-j}-j$. So,

$$
t_{n} \geq t_{k}-(n-k) \forall n \geq k \geq 0 .
$$

Remark 3.3. Note that $\operatorname{dist}\left(U^{1}, M \backslash P_{0}\right)>\delta_{0} \sigma^{n / 2}, \forall U \in \mathcal{U}_{n}\left(P_{0}\right)$ and $n \geq 1$. In particular,

$$
U^{1} \subset P_{0}, \forall U \in \mathcal{U}_{n}\left(P_{0}\right) .
$$

To check the remark 3.3. first observe that $\operatorname{dist}\left(\mathbb{A}_{n-1}\left(P_{0}\right), M \backslash P_{0}\right) \geq$ $\delta_{0} \sigma^{(n-1) / 2}$ (because $t_{n-1}(x)=0$ implies by remark 3.2 that $t_{0}(x) \leq$ $n-1)$. On the other hand, $U^{1}$ is a pre-image (at a hyperbolic time) of some set $B_{\delta_{0}}(P)(P \in \mathcal{P})$ with diameter smaller than $3 \delta_{0}$, and so the diameter of $U^{1}$ is smaller than $3 \delta_{0} \sigma^{n / 2}$. Since $U^{1} \cap \mathbb{A}_{n-1}\left(P_{0}\right) \neq \emptyset$, we get $\operatorname{dist}\left(U^{1}, M \backslash P_{0}\right) \geq \delta_{0} \sigma^{(n-1) / 2}-3 \delta_{0} \sigma^{n / 2}=\delta_{0} \sigma^{n / 2}\left(1 / \sigma^{1 / 2}-3\right)>\delta_{0} \sigma^{n / 2}$.

At this point we have completely described the inductive construction restricted to $P_{0}$ of the sets $\mathbb{A}_{n}\left(P_{0}\right), \mathbb{B}_{n}\left(P_{0}\right)$ and $\Delta_{n}\left(P_{0}\right)$. Proceeding in the same way to the other elements of $\mathcal{P}$ we obtain

$$
\begin{aligned}
\mathbb{A}_{n} & =\bigcup_{P \in \mathcal{P}} \mathbb{A}_{n}(P), \\
\mathbb{B}_{n} & =\bigcup_{P \in \mathcal{P}} \mathbb{B}_{n}(P) \\
\Delta_{n} & =\bigcup_{P \in \mathcal{P}}^{\text {and }} \Delta_{n}(P) .
\end{aligned}
$$

Let $M^{*}=\left\{x \in M \| f^{n}(x) \in \Delta_{0} \forall n \geq 0\right\}$. As $f$ is a diffeomorphism Lebesgue almost everywhere, we have $\operatorname{Leb}\left(M \backslash M^{*}\right)=0$. Finally let us define a time function

$$
R: M^{*} \backslash \bigcap_{n} \Delta_{n} \rightarrow \mathbb{N}
$$

setting $R(x)=n$ when $x \in \Delta_{n-1} \backslash \Delta_{n}$.

We may think of the set $\mathbb{A}_{n-1}\left(\left\{t_{n-1}=0\right\} \cap \Delta_{n-1}\right)$ as the set of allowed points of the $n$-th step of the construction, that is, the set of points $x \in M$ that can be used in the step $\mathrm{n}$, if $n$ is a hyperbolic time to $x$, as the "center" of a new element of the partition (a component of $\{R=n\})$. On the other hand, the set $\mathbb{B}_{n-1}\left(\left\{t_{n-1}>0\right\} \cap \Delta_{n-1}\right)$ is the forbidden points of the $n$-th step, i.e., if $x \in \mathbb{B}_{n-1}$ then, though $x$ does not belong to a constructed element $(x \notin\{R \leq n-1\})$, we can not use $x$ to be the "center" of a new element, even if $x \in H_{n}$. In fact, $\mathbb{B}_{n-1}$ is the intersection of $\Delta_{n-1}$ with an union of "protection 
collars" associated to the components of $\{R \leq n-1\}$ and we can not use the points of $\mathbb{B}_{n-1}$ in the step $n$ because at this time the pre-image of the elements of the triangulation associated to some hyperbolic preball $V_{n}(x)$ (with $x \in H_{n}$ ) may be big compared with these collars and, probably, there will be overlaps of this pre-image with some element constructed already.

Remark 3.4. It is important to emphasize that we only constructed a new element of the partition as a pre-image, associated to a hyperbolic time, of some element of the triangulation. That is, if $U$ is a component of $\{R=n\}$, for some $n$, then $U \subset V_{n}(x)$ for some $x \in \mathbb{A}_{n-1} \cap H_{n}$. As a consequence, $U$ is diffeomorphically mapped, with bounded distortion and good properties of expansion (see proposition [2.5), to some element of the triangulation. This control of distortion and expansion is fundamental to obtain the properties required in the piecewise uniformly expanding Markovian map associated to the Markov structure.

3.3. Collars and rings. To make precise the meaning of "collar", we introduce some additional notation. Given $P_{0} \in \mathcal{P}$ we define, for any $n \geq 0$, the $n$-th former collar of $P_{0}$, or the $(0, n)$-collar of $P_{0}$ as the set

$$
C_{0, n}\left(P_{0}\right)=\left\{x \in P_{0} \| \operatorname{dist}\left(x, \partial P_{0}\right)<\delta_{0} \sigma^{n / 2}\right\},
$$

that is,

$$
C_{0, n}\left(P_{0}\right)=\left\{t_{0} \geq n+1\right\} \cap P_{0} .
$$

For any element $U \in \mathcal{U}_{k}\left(P_{0}\right)$ (a connected component of $\{R=k\}$ ) and each $n \geq k$, we define the $(k, n)$-th collar of $P_{0}$ associated to $U$ as

$$
C_{k, n, U}\left(P_{0}\right)=\bigcup_{j=n-k+1}^{\infty} U^{1}(j) .
$$

A collar of the $n$-th step of the construction is any $(k, n)$-th collar, with $k \leq n$. The collection of all collars of the $n$-th step associated to $P_{0}$ is denoted by $\beta_{n}\left(P_{0}\right)$, i.e.,

$$
\beta_{n}\left(P_{0}\right)=\left\{C_{0, n}\left(P_{0}\right)\right\} \cup\left\{C_{k, n, U}\left(P_{0}\right) \| U \in \mathcal{U}_{k}\left(P_{0}\right) \text { and } 1 \leq k \leq n\right\} .
$$

Finally, we define $C_{k, n, U}^{\prime}\left(P_{0}\right)$, the external ring of the collar $C_{k, n, U}$ $\left(P_{0}\right)$, as

$$
C_{k, n, U}^{\prime}\left(P_{0}\right)=\underbrace{C_{k, n, U}\left(P_{0}\right) \backslash C_{k, n+1, U}\left(P_{0}\right)}_{U^{1}(n-k+1)}
$$

and the external ring of the former collar $C_{0, n}\left(P_{0}\right)$ as

$$
C_{0, n}^{\prime}\left(P_{0}\right)=\underbrace{C_{0, n}\left(P_{0}\right) \backslash C_{0, n+1}\left(P_{0}\right)}_{C_{0, n}\left(P_{0}\right) \cap\left\{t_{0}=n+1\right\}} .
$$


We claim that

$$
\bigcup_{Q \in \beta_{n}} Q=\left\{t_{n} \geq 1\right\} \quad \text { and } \bigcup_{Q \in \beta_{n}} Q \backslash Q^{\prime}=\left\{t_{n}>1\right\} \quad \forall n \in \mathbb{N} \text {, }
$$

where $\beta_{n}=\bigcup_{P \in \mathcal{P}} \beta_{n}(P)$ and $Q^{\prime}$ is denoting the external ring of the collar $Q$. Indeed, if $x \in C_{0, n}\left(P_{0}\right)=\left\{t_{0} \geq n+1\right\} \cap P_{0}$, it follows form Remark 3.2 that $t_{n}(x) \geq 1$. On the other hand, if $x \in C_{k, n, U}\left(P_{0}\right)$, then $x \in U^{1}(j)$ for some $j \geq n-k+1$. Hence, $t_{n}(x) \geq t_{k}(x)-$ $(n-k) \geq t_{k}^{\prime}(x)-(n-k) \geq j-(n-k) \geq 1$. In the same way, if $x \in C_{0, n}\left(P_{0}\right) \backslash C_{0, n}^{\prime}\left(P_{0}\right)$ or $C_{k, n, U}\left(P_{0}\right) \backslash C_{k, n, U}^{\prime}\left(P_{0}\right)$ we get $t_{n}(x)>1$. So, $\bigcup_{Q \in \beta_{n}} Q \subset\left\{t_{n} \geq 1\right\}$ and $\bigcup_{Q \in \beta_{n}} Q \backslash Q^{\prime} \subset\left\{t_{n}>1\right\}$. Now, let us assume that $x \in P_{0}$ and $t_{n}(x)=j \geq 1$. Note that $t_{n}=\max \left\{t_{n}^{\prime}, t_{n-1}-1\right\}$ $=\max \left\{t_{n}^{\prime}, \max \left\{t_{n-1}^{\prime}, t_{n-2}-1\right\}-1\right\}=\max \left\{t_{n}^{\prime}, t_{n-1}^{\prime}-1, t_{n-2}-2\right\}=$ $\max \left\{t_{n}^{\prime}, t_{n-1}^{\prime}-1, t_{n-2}^{\prime}-2, t_{n-3}-3\right\}=\ldots$ Thus,

$$
t_{n}=\max \left\{t_{n}^{\prime}, t_{n-1}^{\prime}-1, t_{n-2}^{\prime}-2, \ldots, t_{1}^{\prime}-(n-1), t_{0}-n\right\}
$$

and $t_{n}(x)=j \geq 1$ implies that $t_{0}(x)=j+n$ or $t_{k}^{\prime}(x)=j+(n-k)$, for some $1 \leq k \leq n$. In the first case we get $x \in C_{0, n}\left(P_{0}\right)$ (and $x \in C_{0, n}^{\prime}\left(P_{0}\right)$ when $j=1)$. In the second one, by the definition of $t_{k}^{\prime}$, there exists $U \in \mathcal{U}_{k}\left(P_{0}\right)$ such that $x \in U^{1}(n-k+j)$. So, $x \in C_{k, n, U}\left(P_{0}\right)$ (and $x \in$ $C_{k, n, U}^{\prime}\left(P_{0}\right)$ only if $\left.j=1\right)$. In any case, we obtain $\left\{t_{n} \geq 1\right\} \subset \bigcup_{Q \in \beta_{n}} Q$ (and also $\left\{t_{n}>1\right\} \subset \bigcup_{Q \in \beta_{n}} Q \backslash Q^{\prime}$ ), thus proving the claim.

The next lemma is important for preventing the overlaps on the sets of the partition. Indeed, in the $n$-th step of the induction, associated to each element $V \in \mathcal{U}_{k}\left(P_{0}\right)$ already constructed ( $V$ is a connected component of $\{R=k\}$, for some $0<k<n)$, there is a collar $Q=C_{k, n, V}\left(P_{0}\right) \in \beta_{n}\left(P_{0}\right)$ around it. By the lemma below, the new components cannot intersect "too much" the collar $Q$. More precisely, if $U \in \mathcal{U}_{n}\left(P_{0}\right)$ (i.e., $U$ is a connected component of $\{R=n\}$ ) then the possible intersection of $U$ with $Q$ will be necessarily within the external ring $Q^{\prime}=C_{k, n, V}^{\prime}\left(P_{0}\right)$. As a consequence, according to Corollary [3.6. there will be no overlaps.

Lemma 3.5. If $U \in \mathcal{U}_{n}\left(P_{0}\right)$ then $U \cap\left\{t_{n-1}>1\right\}=\emptyset$.

Proof. Let us assume that $U \cap\left\{t_{n-1}>1\right\} \neq \emptyset$ for some $U \in \mathcal{U}_{n}\left(P_{0}\right)$. The set $U$ can be written as $U=\left(\left.f^{n}\right|_{V_{n}\left(x_{1}\right)}\right)^{-1}\left(P_{1}\right)$, where $x_{1}$ is some point in $\mathbb{A}_{n-1} \cap H_{n}$ and $P_{1}$ is an element of the triangulation $\mathcal{P}$. By (12), we have

$$
U \cap\left(Q \backslash Q^{\prime}\right) \neq \emptyset \text { for some collar } Q \in \beta_{n-1}\left(P_{0}\right),
$$

where $Q^{\prime}$ is its external ring. Firstly let us verify that $Q$ is not the $(n-1)$-th former collar, i.e, $Q \neq C_{0, n-1}\left(P_{0}\right)$. For this, let us suppose 
by contradiction that $Q=C_{0, n-1}\left(P_{0}\right)$. It follows from Remark 3.3 that $\operatorname{dist}\left(U, \partial P_{0}\right)>\delta_{0} \sigma^{n / 2}$ and, as a consequence, $t_{0}(x) \leq n \forall x \in U$. So, $U \cap\left(Q \backslash Q^{\prime}\right)=U \cap\left(C_{0, n-1}\left(P_{0}\right) \backslash C_{0, n-1}^{\prime}\left(P_{0}\right)\right)=U \cap\left\{t_{0}>n\right\} \cap P_{0}=\emptyset$, thus contradicting (14).

As $Q$ is not the $(n-1)$-th former collar, there exist $1 \leq k \leq n-1$ and $V \in \mathcal{U}_{k}\left(P_{0}\right)$ such that $Q=C_{k, n-1, V}\left(P_{0}\right) \in \beta_{n-1}\left(P_{0}\right)$. On its turn, $V$ can be written as $\left(\left.f^{k}\right|_{V_{k}\left(x_{2}\right)}\right)^{-1}\left(P_{2}\right)$, where $x_{2}$ is some point of $\mathbb{A}_{k-1} \cap H_{k}$ and $P_{2} \in \mathcal{P}$.

By construction, $f^{k}$ sends diffeomorphically $V$ onto $P_{2}, V \cup Q$ onto the topological ball $B_{\delta_{0} \sigma^{(n-1-k) / 2}}\left(P_{2}\right)$ and $Q^{\prime}$ onto the "ring" $I_{n-k}^{1}\left(P_{2}\right)=$ $B_{\delta_{0} \sigma^{(n-1-k) / 2}}\left(P_{2}\right) \backslash B_{\delta_{0} \sigma^{(n-k) / 2}}\left(P_{2}\right)$. Thus, $Q^{\prime}$ splits $M$ into two connected components, that is, taking $W=V \cup\left(Q \backslash Q^{\prime}\right)$ and $L=M \backslash(V \cup Q)$, we get $M \backslash Q^{\prime}=W \cup L$ with $W \cap L=\emptyset$. Note that $W \supset V$ and $L \supset \mathbb{A}_{n-1}$ (because $V \cap \mathbb{A}_{j} \subset V \cap \Delta_{j}=\emptyset \forall j \geq k$ and, as $t_{k}(x) \geq t_{k}^{\prime}(x) \geq n-k$ $\forall x \in Q$, it follows from remark 3.2 that $t_{n-1}(x) \geq n-k-(n-1-k)=$ $1>0 \forall x \in Q)$.

We know that $U \cap \mathbb{A}_{n-1} \neq \emptyset$, because, by construction, $x_{1} \in \mathbb{A}_{n-1} \cap$ $H_{n}$. Hence, $U$ intersects $L$. On the other hand, we are assuming that $U \cap W \supset U \cap\left(Q \backslash Q^{\prime}\right) \neq \emptyset$. So, it follows from the connectivity of $U$ that it not only intersects $Q^{\prime}$ but also intersects both connected components of the boundary of $Q^{\prime}$ (recall that $f^{n}$ sends diffeomorphically $\partial Q^{\prime}$ onto $\left.\partial I_{n-k}^{1}\left(P_{2}\right)=\partial B_{\delta_{0} \sigma^{(n-1-k) / 2}}\left(P_{2}\right) \cup \partial B_{\delta_{0} \sigma^{(n-k) / 2}}\left(P_{2}\right)\right)$.

Therefore, we can pick two points in $U, q_{1}$ and $q_{2}$, in distinct components of the boundary of $Q^{\prime}$. We assume that $f^{k}\left(q_{1}\right) \in \partial B_{\delta_{0} \sigma^{(n-1-k) / 2}}\left(P_{2}\right)$ and $f^{k}\left(q_{2}\right) \in \partial B_{\delta_{0} \sigma^{(n-k) / 2}}\left(P_{2}\right)$. As $U$ is the pre-image of $P_{1}$ in a hyperbolic time, we get from Proposition 2.5 that

$$
\operatorname{dist}\left(f^{k}\left(q_{1}\right), f^{k}\left(q_{2}\right)\right) \leq \sigma^{(n-k) / 2} \operatorname{dist}\left(f^{n}\left(q_{1}\right), f^{n}\left(q_{2}\right)\right)<\delta_{0} \sigma^{(n-k) / 2} .
$$

On the other hand,

$$
\begin{aligned}
\operatorname{dist}\left(f^{k}\left(q_{1}\right), f^{k}\left(q_{2}\right)\right) & \geq \delta_{0} \sigma^{(n-1-k) / 2}-\delta_{0} \sigma^{(n-k) / 2} \\
& =\delta_{0} \sigma^{(n-k) / 2}\left(\sigma^{-1 / 2}-1\right)
\end{aligned}
$$

which combined with (15) gives $\sigma>\frac{1}{4}$. This contradicts our choice of $\sigma($ see (7) $)$.

Corollary 3.6. If $U$ and $V$ are distinct components of $\{R=n\}$ and $\{R=m\}$ then $U \cap V=\emptyset$

Proof. Suppose that $U=\left(\left.f^{n}\right|_{V_{n}\left(x_{1}\right)}\right)^{-1}\left(P_{1}\right)$ and $V=\left(\left.f^{m}\right|_{V_{m}\left(x_{2}\right)}\right)^{-1}\left(P_{2}\right)$, with $P_{1}$ and $P_{2} \in \mathcal{P}$. By Remark 3.3 we can assume that both $U$ and $V$ are contained in some $P_{0} \in \mathcal{P}$. As we claimed during the presentation of the algorithm, if $n=m$ we have $U \cap V=\emptyset$. So, let us assume that 
$m<n$. Let $Q=C_{m, n-1, V}\left(P_{0}\right)$ be the $(n-1)$-th collar associated to $V$ and $Q^{\prime}=C_{m, n-1, V}^{\prime}\left(P_{0}\right)$ its external ring.

By construction, $f^{m}$ sends diffeomorphically $V$ onto the topological ball $P_{2}$ and the set $T=Q \backslash Q^{\prime}$ diffeomorphically onto the "ring" $B_{\delta_{0} \sigma^{(n-m) / 2}}\left(P_{2}\right) \backslash P_{2}=\bigcup_{k=n-m+1}^{\infty} I_{k}^{1}\left(P_{2}\right)$. Thus, $T$ splits $M$ into two connected components. Indeed, taking $L=M \backslash(V \cup T)$, we get $M \backslash T=V \cup L$ with $V \cap L=\emptyset$.

It is easy to see that $T \subset\left\{t_{n-1}>1\right\}$. In fact, as $t_{m}(x) \geq t_{m}^{\prime}(x) \geq n-$ $m+1 \forall x \in T\left(=Q \backslash Q^{\prime}=V^{1}((n-1)-m+2) \cup V^{1}((n-1)-m+3) \cup \ldots\right)$, it follows from Remark 3.2 that $t_{n-1}(x) \geq n-m+1-(n-1-m)=2$ $\forall x \in T$.

Note that $L \supset \mathbb{A}_{n-1}$ (because $V \cap \mathbb{A}_{j} \subset V \cap \Delta_{j}=\emptyset \forall j \geq m$ and $\left.\mathbb{A}_{n-1} \cap T \subset \mathbb{A}_{n-1} \cap\left\{t_{n-1} \neq 0\right\}=\emptyset\right)$. We know that $U \cap \mathbb{A}_{n-1} \neq \emptyset$, because by construction $x_{1} \in \mathbb{A}_{n-1} \cap H_{n}$. Hence, $U$ intersects $L$. If $U \cap V \neq \emptyset$, it follows from the connectivity of $U$ that $U \cap T \neq \emptyset$. But this contradicts Lemma 3.5. as $T \subset\left\{t_{n-1}>1\right\}$.

The proposition below says that the sum of the Lebesgue measure of all forbidden sets is finite. This finiteness is fundamental to assure that almost every point in $M$ belongs to a constructed element, i.e., $\operatorname{Leb}\left(M \backslash \bigcup_{n}\{R=n\}\right)=0$.

Proposition 3.7. $\sum_{n=0}^{\infty}$ Leb $\mathbb{B}_{n}<\infty$.

Proof. First of all, we compare the Lebesgue measure of the $(k, n)$-th collar $C_{k, n, U}\left(P_{0}\right)$ with the measure of $U \in \mathcal{U}_{k}\left(P_{0}\right)$, where $P_{0}$ is an element of $\mathcal{P}$ and $1 \leq k \leq n$. For this, recall that there must be some hyperbolic pre-ball $V_{k}\left(x_{0}\right)$ (containing $U$ and $C_{k, n, U}\left(P_{0}\right)$ ) and an element $P_{1}$ of $\mathcal{P}$ such that $\left.f^{k}\right|_{V_{k}\left(x_{0}\right)}$ maps $U$ diffeomorphically onto $P_{1}$ and $C_{k, n, U}\left(P_{0}\right)$ onto $\bigcup_{j=n-k+1}^{\infty} I_{j}^{1}\left(P_{1}\right)$, both with the distortion bounded by an universal constant $C_{1}=\exp (\rho \operatorname{diameter}(M))$ (see proposition 2.5). Thus, it is sufficient to compare the measures of $\bigcup_{j=n-k+1}^{\infty} I_{j}^{1}\left(P_{1}\right)$ and $P_{1}$. As $P_{1} \in \mathcal{P}$ is the interior of an element of a triangulation of $M$, the boundary of $P_{1}$ is a finite union of smooth codimension one submanifolds. So, for $r>0$ small we have $\operatorname{Leb}\left(B_{r}\left(P_{1}\right) \backslash P_{1}\right) \approx r$. As $\mathcal{P}$ is a finite collection, one can find $\xi>0$ such that

$$
\frac{\operatorname{Leb}\left(B_{r}(P) \backslash P\right)}{\operatorname{Leb} P}<\xi r \text { for all } P \in \mathcal{P} \text { and } r>0 \text {. }
$$

Hence,

$$
\begin{aligned}
& \frac{\operatorname{Leb} C_{k, n, U}\left(P_{0}\right)}{\operatorname{Leb} U} \leq C_{1} \frac{\operatorname{Leb}\left(\bigcup_{j=n-k+1}^{\infty} I_{j}^{1}\left(P_{1}\right)\right)}{\operatorname{Leb} P_{1}}= \\
& =C_{1} \frac{\operatorname{Leb}\left(B_{\delta_{0} \sigma^{n-k}}\left(P_{1}\right) \backslash P_{1}\right)}{\operatorname{Leb} P_{1}}<C_{1} \xi \delta_{0} \sigma^{n-k} .
\end{aligned}
$$


Thus, setting $C_{2}=C_{1} \xi \delta_{0}$, we have

$$
\text { Leb } C_{k, n, U}\left(P_{0}\right)<C_{2} \sigma^{n-k} \text { Leb } U
$$

for all $1 \leq k \leq n, U \in \mathcal{U}_{n}\left(P_{0}\right)$ and $P_{0} \in \mathcal{P}$.

As in (16), one can find some $\varsigma>0$ such that

$$
\frac{\text { Leb } C_{0, n}(P)}{\text { Leb } P}<\varsigma \sigma^{n} \text { for all } P \in \mathcal{P} \text { and } n \geq 0 \text {. }
$$

Using (17) and (18), it follows that

$$
\begin{gathered}
\sum_{Q \in \beta_{n}\left(P_{0}\right)} \quad \text { Leb } Q=\operatorname{Leb} C_{0, n}\left(P_{0}\right)+\sum_{k=1}^{n} \sum_{U \in \mathcal{U}_{k}\left(P_{0}\right)} \operatorname{Leb} C_{k, n, U}\left(P_{0}\right) \\
<\varsigma \sigma^{n} \text { Leb } P_{0}+\sum_{k=1}^{n} \sum_{U \in \mathcal{U}_{k}\left(P_{0}\right)} C_{2} \sigma^{n-k} \operatorname{Leb} U= \\
=\varsigma \sigma^{n} \text { Leb } P_{0}+C_{2} \sum_{k=1}^{n} \sigma^{n-k} \operatorname{Leb}\left(\{R=k\} \cap P_{0}\right) .
\end{gathered}
$$

Thus,

$$
\begin{gathered}
\sum_{Q \in \beta_{n}} \text { Leb } Q=\sum_{P_{0} \in \mathcal{P}} \sum_{Q \in \beta_{n}\left(P_{0}\right)} \text { Leb } Q< \\
<\varsigma \sigma^{n} \underbrace{\operatorname{Leb} M}_{1}+C_{2} \sum_{k=1}^{n} \sigma^{n-k} \operatorname{Leb}\{R=k\}, \quad \forall n \geq 1 .
\end{gathered}
$$

As

$$
\begin{gathered}
\sum_{n=1}^{\infty} \sum_{k=1}^{n} \sigma^{n-k} \operatorname{Leb}\{R=k\} \leq \operatorname{Leb}\{R=1\}+ \\
+(\sigma \operatorname{Leb}\{R=1\}+\operatorname{Leb}\{R=2\})+ \\
+\left(\sigma^{2} \operatorname{Leb}\{R=1\}+\sigma \operatorname{Leb}\{R=2\}+\operatorname{Leb}\{R=3\}\right)+\ldots= \\
=\sum_{j=0}^{\infty} \sigma^{j} \operatorname{Leb}\{R=1\}+\sum_{j=0}^{\infty} \sigma^{j} \operatorname{Leb}\{R=2\}+\ldots= \\
=\frac{1}{1-\sigma} \sum_{n=1}^{\infty} \operatorname{Leb}\{R=n\} \leq \frac{1}{1-\sigma} \underbrace{\operatorname{Leb} M}_{1},
\end{gathered}
$$

we get

$$
\sum_{n=0}^{\infty} \sum_{Q \in \beta_{n}} \text { Leb } Q=\varsigma+\sum_{n=1}^{\infty} \sum_{Q \in \beta_{n}\left(P_{0}\right)} \text { Leb } Q \leq \varsigma+\varsigma \frac{\sigma}{1-\sigma}+\frac{C_{2}}{1-\sigma}<\infty .
$$


This completes the proof, because $\mathbb{B}_{n}=\bigcup_{Q \in \beta_{n}} Q \cap \Delta_{n}$, and so,

$$
\sum_{n=0}^{\infty} \text { Leb } \mathbb{B}_{n}<\frac{\varsigma+C_{2}}{1-\sigma}<\infty .
$$

Now, we are going to prove that this algorithm does indeed produce a partition Lebesgue mod 0 of $M$. As Corollary 3.6 assures that the elements constructed by this algorithm are mutually disjoint, we have only to check that $\operatorname{Leb}\left(\bigcap_{n} \Delta_{n}\right)=0\left(\Delta_{0} \supset \Delta_{1} \supset \ldots\right.$ is a nested sequence).

To prove this, first observe that, as $\sum_{n=0}^{\infty}$ Leb $\mathbb{B}_{n}<\infty$, it follows from Borel-Cantelli lemma that Lebesgue almost every point in $M$ belongs only to finitely many $\mathbb{B}_{j}{ }^{\prime} s$, that is, defining the function $b$ : $M \rightarrow \mathbb{N}$ by

$$
b(x)=\left\{\begin{array}{ll}
\#\left\{n \in \mathbb{N} \| x \in \mathbb{B}_{n-1}\right\} & \text { if } x \in \Delta_{0} \\
+\infty & \text { if } x \notin \Delta_{0}
\end{array},\right.
$$

we have $b(x)<+\infty$ for Lebesgue almost all $x \in M$ (because $\int b d L e b=$ $\sum_{n}$ Leb $\left.\mathbb{B}_{n}\right)$. Moreover, as any Lebesgue generic point has infinity many hyperbolic times, one can find for almost every $x \in M^{*}$ the first time $n>\max \left\{n \in \mathbb{N} \| x \in \mathbb{B}_{n-1}\right\}$ such that $x \in H_{n}$. In this case, if $x$ still belongs to $\Delta_{n-1}$ we have $x \in \Delta_{n-1} \backslash \Delta_{n}$. In other words, almost every point in $M^{*}$ (and consequently, in $M$ ) belongs to some $\Delta_{n-1} \backslash \Delta_{n}$. So, $\operatorname{Leb}\left(\bigcap_{n} \Delta_{n}\right)=0$.

Markov Structures. Let $F: \Delta^{*} \rightarrow M$ be given by $F(x)=f^{R(x)}(x)$, where $\Delta^{*}=M^{*} \backslash \bigcap_{n} \Delta_{n}$. By construction, the map $F$ is a piecewise uniformly expanding Markovian map (see remark 3.4) and we conclude the proof of the first part of the Main Theorem.

\section{Integrability of The Time Function}

In this section we finish the proof of the Main Theorem showing the integrability of the time function of the Markov Structure constructed in the previous section. We will use the objects and notation of the Partitioning Algorithm. Let $\nu$ be one of the $F$-invariant measure given by the Markov Structure and assume by contradiction that $\left.\sum_{P \in \mathcal{P}} R\right|_{P} \nu(P)=\infty$. It follows from Birkhoff's Ergodic Theorem that

$$
\frac{1}{n} \sum_{j=0}^{n-1} R \circ F^{j}(x) \longrightarrow \int R d \nu=\left.\sum_{P \in \mathcal{P}} R\right|_{P} \nu(P)=\infty,
$$


for $\nu$-almost every point $x \in M$.

As the density of $\nu$ is uniformly bounded from above, it follows from proposition 3.7 that $\sum_{n=0}^{\infty} \nu\left(\mathbb{B}_{n}\right)<\infty$. Hence, for $\nu$-generic points $x \in M$ we get

$$
\frac{1}{n} \sum_{j=0}^{n-1} b \circ F^{j}(p) \longrightarrow \int b d \nu=\sum_{n} \nu\left(\mathbb{B}_{n}\right)<\infty
$$

Let $M^{* *}=\left\{x \in M^{*} \| F^{j}(x) \in \operatorname{Dom}(F) \forall j \geq 0\right\}$. It is clear that $\nu\left(M \backslash M^{* *}\right)=0$. Let $x \in M^{* *}$. Set, for every $i \in \mathbb{N}, j_{i}=j_{i}(x)=$ $\sum_{j=0}^{i-1} R \circ F^{j}(x)$, that is, $F^{i}(x)=f^{j_{i}}(x)$. Given $j \in \mathbb{N}$, there exists a unique integer $s=s(j) \geq 0$ such that $j_{s} \leq j<j_{s+1}$. Let us set $\mathcal{I}_{0}=\left\{j_{1}, j_{2}, j_{3}, \ldots\right\}$ and suppose that $x \in H_{j}$. In this case, $F^{s}(x) \in$ $H_{m}$, where $m=j-j_{s}$. By construction, if $F^{s}(x) \notin\{R=m\}$ (i.e. $\left.m<R\left(F^{s}(x)\right)\right)$, then $F^{s}(x) \in \mathbb{B}_{m-1}$. On the other hand, if $F^{s}(x) \in$ $\{R=m\}$, then $m=j_{s+1}-j_{s}$, which implies $j=j_{s+1} \in \mathcal{I}_{0}$.

As the number of integers $\ell$ between $j_{i}$ and $j_{i+1}$ such that $x \in H_{\ell}$ is bounded by the number of integers $m$ such that $F^{i}(x) \in \mathbb{B}_{m-1}$, we have

$$
\#\left\{\ell \in\left\{j_{i}+1, \ldots, j_{i+1}-1\right\} \| x \in H_{\ell}\right\} \leq b\left(F^{i}(x)\right) .
$$

Thus, for each $n \in \mathbb{N}$ we can write

$$
\begin{gathered}
\#\left\{j \leq n \| x \in H_{j}\right\} \leq \#\left\{j \leq n \| j \in \mathcal{I}_{0}\right\}+\sum_{i=0}^{s(n)} b\left(F^{i}(x)\right) \leq \\
\leq s(n)+\sum_{i=0}^{s(n)} b\left(F^{i}(x)\right)
\end{gathered}
$$

Therefore,

$$
\frac{1}{n} \#\left\{1 \leq j \leq n \| x \in H_{j}\right\} \leq \frac{s(n)}{n}\left(1+\frac{1}{s(n)} \sum_{j=0}^{s(n)} b \circ F^{j}(x)\right)
$$

By construction, if $s(n)=i$, that is, $j_{i} \leq n<j_{i+1}$, then

$$
\frac{j_{i}}{i} \leq \frac{n}{s(n)}<\frac{j_{i+1}}{i+1}\left(1+\frac{1}{i}\right)
$$

As $\frac{j_{i}}{s\left(j_{i}\right)}=\frac{j_{i}}{i}=\frac{1}{i} \sum_{j=0}^{i-1} R \circ F^{j}(x) \rightarrow \infty$, it follows from equations (20) and (21) that

$$
\lim _{n \rightarrow \infty} \frac{1}{n} \#\left\{1 \leq j \leq n \| x \in H_{j}\right\}=0 .
$$


But this is an absurd as one can see in Remark 3.1. So, we necessarily have the time function $R \nu$-integrable and so, we conclude the proof of the Main Theorem.

To prove theorems 1, 22 and 3] let $\left\{\nu_{1}, \ldots, \nu_{s}\right\}$ be the set of ergodic absolutely continuous invariant measures given by Theorem 4 .

Proof of Theorem 1. As we observed before it is straightforward to check that as the time function $R$ is $\nu_{i}$-integrable, then

$$
\mu_{i}=\sum_{j=0}^{\infty} f_{*}^{j}\left(\nu_{i} \mid\{R>j\}\right)
$$

is an ergodic absolutely continuous $f$-invariant measure. Here $\nu_{i} \mid\{R>$ $j\}$ denotes the measure given by $\nu_{i} \mid\{R>j\}(A)=\nu_{i}(A \cap\{R>j\})$, and $f_{*}^{j}$ denotes the push-forward of the measure by $f^{j}$. Moreover, it follows from Theorem 4 that almost every point in $M$ belongs to the basin of one of the $\nu_{i}$. Therefore, almost every point $x \in M$ also belongs to the basin of one of the measures $\mu_{1}, \ldots, \mu_{s}$.

Remark 4.1. In the beginning of the Partitioning Algorithm we could have replaced, if necessary, $f$ by some iterated $f^{n_{0}}$ and so, the measures $\mu_{1}, \ldots, \mu_{s}$ might be only $f^{n_{0}}$ invariant. In such case we consider the induced $f$-invariant measures $\mu_{1}^{\prime}, \ldots, \mu_{s}^{\prime}$ given by

$$
\mu_{i}^{\prime}(A)=\mu_{i}(A)+\mu_{i}\left(f^{-1} A\right)+\ldots+\mu_{i}\left(f^{-\left(n_{0}-1\right)} A\right)
$$

4.1. Proof of Theorem 2. As any non-uniformly expanding map satisfies the hypothesis of Theorem 1 we have only to check that every non-flat map with the slow recurrence satisfying equation (3) for almost every $x \in M$ also satisfies equation (2). This result is a consequence of the integrability of $x \mapsto \log \left\|(D f(x))^{-1}\right\|$ with respect to any absolutely continuous invariant measure ( see Lemma 4.2 below ). Indeed, as $\log \left\|(D f)^{-1}\right\|^{-1}=-\log \left\|(D f)^{-1}\right\|$, if $\log \left\|(D f)^{-1}\right\| \in L^{1}\left(\mu_{i}\right)$ it follows from Birkhoff's Ergodic Theorem that we have for $\mu_{i}$ almost all $x \in M$

$$
\begin{aligned}
& \liminf _{n \rightarrow \infty} \frac{1}{n} \sum_{j=0}^{n-1} \log \left\|\left(D f\left(f^{n}(x)\right)\right)^{-1}\right\|^{-1}= \\
& =\limsup _{n \rightarrow \infty} \frac{1}{n} \sum_{j=0}^{n-1} \log \left\|\left(D f\left(f^{n}(x)\right)\right)^{-1}\right\|^{-1} .
\end{aligned}
$$

As Lebesgue almost every point $x \in M$ belongs to the basin of some $\mu_{i}$, we conclude that the equality above of lim inf and lim sup holds for Lebesgue almost all $x \in M$. 
Lemma 4.2. If $f: M \rightarrow M$ is a $C^{2}$ endomorphism, then $\log \left\|(D f)^{-1}\right\|$ is integrable with respect to any absolutely continuous invariant measure.

Proof. In 12] (see remark 1.2 of [12]) it was proved that

$$
-\infty<\int \log |\operatorname{det} D f| d \mu<+\infty
$$

with respect to any absolutely continuous invariant measure $\mu$. Let us show how this result implies the integrability of $\log \left\|(D f)^{-1}\right\|$. It is easy to see that $|\lambda| \leq\|A\|$ for any eigenvalue $\lambda$ of a matrix $A \in \mathbb{R}^{m \times m}$. Thus, $|\operatorname{det} A| \leq\|A\|^{m}$ and so

$$
-\infty<\frac{1}{\operatorname{dim}(M)} \int \log |\operatorname{det} D f| d \mu \leq \int \log \|D f\| d \mu<+\infty .
$$

We know that $\operatorname{adj}(A) A=\operatorname{det}(A) I \forall A \in \mathbb{R}^{n \times n}$, where adj $A$ is the (classical) adjoint matrix, the transposed matrix of cofactors [10], and $I$ is the identity matrix. So, $\log |\operatorname{det} D f|-\log \|D f\| \leq \log \|\operatorname{adj} D f\|$ and so $-\infty<\int \log \|\operatorname{adj} D f\| d \mu$. Moreover, as $\|$ adj $D f \|$ is bounded, we get

$$
-\infty<\int \log \|\operatorname{adj} D f\| d \mu<+\infty .
$$

Finally, from $(D f)^{-1}=\frac{1}{\operatorname{det} D f} a d j D f$ we get

$$
\begin{gathered}
-\infty<\int \log \left\|(D f)^{-1}\right\| d \mu= \\
=\int \log \|\operatorname{adj} D f\| d \mu-\int \log |\operatorname{det} D f| d \mu<+\infty .
\end{gathered}
$$

4.2. Proof of Theorem [3. Suppose that there exists $0<\sigma<1$ and $\delta>0$ such that Lebesgue almost every point $x \in M$ has a $(\sigma, \delta)$ hyperbolic time. So, we have $\operatorname{Leb}(U)=0$, where $U=M \backslash \bigcup_{j} H_{j}(\sigma, \delta)$. Given a Lebesgue generic point $x \in M$ we have $f^{j}(x) \notin U \forall j$. Let $n_{1}$ be such that $x \in H_{n_{1}}(\sigma, \delta)$ and $m_{1}$ such that $f^{n_{1}}(x) \in H_{m_{1}}(\sigma, \delta)$. By the properties of hyperbolic times we get $x \in H_{n_{2}}(\sigma, \delta)$, where $n_{2}=$ $n_{1}+m_{1}>n_{1}$. Therefore, by induction, one concludes that Lebesgue almost every point in $M$ has infinitely many $(\sigma, \delta)$-hyperbolic times. Remembering that $x \in H_{j}(\sigma, \delta)$ implies $\prod_{k=0}^{j-1}\left\|\left(D f \circ f^{k}(x)\right)^{-1}\right\| \leq \sigma^{j}$, 
we have, for generic $x \in M, \frac{1}{j} \sum_{k=0}^{j-1} \log \left\|\left(D f\left(f^{k}(x)\right)\right)^{-1}\right\| \leq \log \sigma<0$ for infinitely many integers $j>0$. Therefore,

$$
\liminf _{j \rightarrow \infty} \frac{1}{j} \sum_{k=0}^{j-1} \log \left\|\left(D f\left(f^{k}(x)\right)\right)^{-1}\right\| \leq \log \sigma<0
$$

for Lebesgue almost all $x \in M$. As the critical set is empty, the map $f$ satisfies the hypothesis of the theorem 11. So, by the theorem 2, $f$ is a non-uniformly expanding map.

Remark 4.3. Using the same argument as in the proof of Theorem 3 , it is easy to conclude that we can change, in the hypothesis of Theorem 3, the existence of a hyperbolic time by the existence of a time with good average of expansion. That is, if $f: M \rightarrow M$ is $C^{2}$ local diffeomorphism and $\exists \lambda>0$ such that for Lebesgue almost every point $x \in M$ one can find at least one $n_{0}=n_{0}(x) \in \mathbb{N}$ such that

$$
\frac{1}{n_{0}} \sum_{j=0}^{n_{0}-1} \log \left(\left\|\left(D f\left(f^{j}(x)\right)\right)^{-1}\right\|^{-1}\right) \geq \lambda>0,
$$

then $f$ is a non-uniformly expanding map.

\section{REFERENCES}

[1] J. Aaronson, An introduction to infinite ergodic theory. Math. Surv. Monographs 50, AMS, Providence R.I. US (1997).

[2] J. F. Alves, SRB measures for non-hyperbolic systems with multidimensional expansion, Ann. Scient. Éc. Norm. Sup., $4^{e}$ série, 33 (2000), 1-32.

[3] J. F. Alves, V. Araújo, Random perturbations of nonuniformly expanding maps, Astérisque 286 (2003), 25-62.

[4] J. F. Alves, C. Bonatti, M. Viana, SRB measures for partially hyperbolic systems whose central direction is mostly expanding, Invent. Math. 140 (2000), 351-398.

[5] J. F. Alves, S. Luzzatto, V. Pinheiro, Markov structures and decay of correlations for non-uniformly expanding dynamical systems, Ann. I. H. Poincaré AN 22 (2005) 817-839.

[6] J. F. Alves, M. Viana, Statistical stability for robust classes of maps with nonuniform expansion, Ergod. Th. \& Dynam. Sys. 22 (2002), 1-32.

[7] M. Benedicks, L.-S. Young, Markov extensions and decay of correlations for certain Hénon maps, Astérisque 261 (2000), 13-56.

[8] W. de Melo, S. van Strien, One Dimensional Dynamics, Springer-Verlag, 1993.

[9] S. Gouezel, Decay of correlations for nonuniformly expanding systems, Bulletin de la Société Mathématique de France (to appear).

[10] R.A. Horn, C.R. Johnson, Matrix Analysis, Cambridge Univ. Press(1990).

[11] G. Keller, Exponents, attractors and Hopf decompositions for interval maps. Ergod. Th. \& Dynam. Sys. (1990), 10, 717-744. 
[12] P.-D. Liu, Pesin's entropy formula for endomorphisms. Nagoya Math. J. Vol. 150 (1998), 197-209.

[13] M. Viana, Multidimensional non-hyperbolic attractors, Publ. Math. IHES 85 (1997), 63-96.

[14] L.-S. Young, Statistical properties of dynamical systems with some hyperbolicity, Ann. Math. 147 (1998), 585-650.

[15] L.-S. Young, Recurrence times and rates of mixing, Israel J. Math. 110 (1999), 153-188.

Departamento de Matemática, Universidade Federal da Bahia, Av. Ademar de Barros s/n, 40170-110 Salvador, Brazil.

E-mail address: viltonj@ufba.br 